\title{
Factores de mayor incidencia en la adopción de innovaciones tic a escala de pais. Importancia de las relaciones directas e indirectas entre factores ${ }^{1}$
}

\author{
Rosa Maria Jordá-Borrella (a), Javier López-Otero (b), Gustavo Contreras-Cabrera (c) \\ (a) Centro de trabajo, Universidad Sevilla, email borrell@us.es \\ (b) Centro de trabajo, Universidad Isabel I, email javier.lopez.otero@ui1.es \\ (c) Centro de trabajo, Universidad Sevilla, email gcontreras@us.es
}

\begin{abstract}
Resumen
Esta investigación modelizó los factores que influyen en la adopción de innovaciones TIC en las empresas (ICT), determinando su influencia directa e indirecta, y la importancia de cada constructo en el modelo. Se identificaron los factores incidentes en la adopción de ICT mediante el modelo TOE. Las hipótesis se testaron usando una muestra de empresas con información agregada a nivel de país, y se empleó PLS-SEM como método de estimación y comprobación de las hipótesis. Posteriormente, se emplearon los algoritmos Betweenness, y Beta Centrality para determinar el papel de cada factor en la red. El constructo más determinante fue la Globalización de las firmas debido a la influencia indirecta de éste en el modelo. Igualmente, resultó clave el constructo del entorno tecnológico ICT por su gran influencia sobre la adopción de innovaciones, así como por su papel transmisor de los efectos indirectos de los demás constructos.
\end{abstract}

Palabras claves: Adopción Tecnologías TIC, Modelo TOE (Tecnología-Entorno-Empresa), Globalización, PLS-SEM, Análisis de Redes.

\section{INTRODUCTION}

En los últimos años se ha producido un creciente interés por las tecnologías ICT o TIC y su impacto en las empresas debido a los efectos que genera en la productividad (Oliveira et al.,2014; Buyya et al., (2009); Škrinjar et al., 2010), internacionalización (Laursen and Salter, 2006), competitividad, reducción de costes y satisfacción del cliente (Ifinedo, 2011; Capó -Vicedo, 2007).

Durante las últimas décadas, las tecnologías ICT han innovado los procesos productivos a causa de la automatización. Y esa transformación de los procesos productivos ha generado a su vez una mejora en la eficiencia de éstos y un incremento de la internacionalización de la empresa. De forma que la simbiosis de ambos procesos ha dado lugar a la globalización de los procesos de producción (Milberg and Winkler (2013).

Precisamente, el concepto de la globalización de los procesos de producción se apoya en los avances tecnológicos, en la capacidad de los países por atraer inversión directa extranjera, en cómo éstos territorios interrelacionan dichas inversiones con el tejido productivo local y en el surgimiento de la sociedad del conocimiento; constituyendo factores fundamentales para que las naciones (Frenz and Letto-Gillies,2009) desarrollen habilidades para internacionalizarse conectándose a redes globales.

Por otra parte, las tecnologías ICT han posibilitado la comunicación empresarial Business to Business (B2B) al contribuir significativamente a la sincronización productiva con proveedores y otras organizaciones (Armbrust et al., 2010) de distintos países, así como a la sincronización de la actividad global de las multinacionales, reforzando su papel en la globalización (Palafox, 2017). Igualmente, el eCommerce ha favorecido el acceso a clientes remotos reduciendo los costes de la puesta en contacto (Mayer et al., 2013 Hong and Zhu, 2006; Oliveira and Martins, 2010).

Paralelamente a la introducción de estas tecnologías se ha producido una creciente disparidad entre las empresas que las adoptan y las que no (Bach et al, 2013), y un incremento de los flujos de inversión directa extran-

\footnotetext{
${ }^{1}$ Este trabajo forma parte del Proyecto Investigación del Ministerio Economía y Competitividad: La brecha digital en la UE, modelos explicativos,
} predictivos y espaciales (CS2015-67662-P). 
jera desde los países avanzados a determinadas naciones emergentes (Chaminade,2012); generando la interrelación de ambos procesos un aumento de la disparidad digital entre los estados a nivel mundial (Alderete et al.,2014; Addison, and Heshmati, (2003). Ante esta nueva situación, los gobiernos se están preocupado por mitigar las diferencias digitales entre países mediante políticas de fomento y difusión de las tecnologías ICT en la empresa y en la administración (Addison, and Heshmati, (2003).

Sin embargo, el sector no ha parado de crecer y se encuentra en una dinámica en la que están surgiendo nuevas tecnologías que pueden transformar de un modo radical los sistemas de producción actuales (Lin, and Lin, 2008; Tsai et al., 2010). En este sentido, la aparición de nuevas tecnologías ICT (Wang and Wang, 2016) conducirán a cambios profundos en los actuales procesos de producción.

En consecuencia, la literatura científica ha dedicado grandes esfuerzos para analizar los mecanismos que influyen en la adopción de ICT en las firmas. Una parte significativa de los trabajos se han centrado en factores relativos a la organización y la tecnología a adoptar (Oliveira et al.; Alderete et al, 2014), destacando los costes de adopción, la fiabilidad y la seguridad (Gupta et al., 2013), o la falta de destrezas para implementar la tecnología. Sin embargo, son pocos los estudios que hayan realizado un análisis integral, tomando como unidad de análisis el país, que incluya aspectos del entorno científico y económico de las naciones, la influencia que ejerce la globalización en las firmas y en los estados adoptantes de ICT, así como la capacidad de gestión de la innovación en las empresas.

De este modo, en esta investigación se abordan los siguientes objetivos: 1.- modelizar a través de las técnicas PLS ( Partial Least Squares) y SEM (Structural Ecuation Modeling) los factores que influyen en la adopción de las innovaciones ICT, empleando como marco de referencia el modelo TOE (tecnología-entorno-empresa). 2. En dicha modelización se identificarán los efectos directos de los constructos elaborados sobre la adopción ICT, así como los factores que inciden de un modo indirecto sobre la adopción de innovaciones ICT.

\section{MODELO DE INVESTIGACIÓN Y DESARROLLO DE HIPÓTESIS}

La adopción de innovaciones en la empresa es un fenómeno ampliamente estudiado en la literatura científica, sin embargo, existe mucha menos investigación dirigida al análisis de la adopción de innovaciones ICT en los países.

Existen varios modelos que abordan la difusión de las innovaciones ICT, entre ellos están TecnologíaEmpresa-Entorno (TOE) (Tornatzky et al., 1990) y el modelo DOI de Rogers (1995). Si bien, de acuerdo con las características de esta investigación resulta más apropiado utilizar el modelo TOE.

Para ello, se ha considerado al país, como un activo relacional o espacio relacional en construcción permanente en función de la globalización, de la dimensión económica-científica de cada estado, del contexto ICT y de la capacidad de las firmas para adoptar ICT. Después se han adaptado los tres constructos TOE: factor del entorno empresarial, factor tecnológico y factor empresarial del problema estudiado.

El factor entorno se concreta según (Tornatzky et al., 1990) en el entorno de las firmas, las características del sector, el grado de competición de las empresas, acceso a recursos de los proveedores y negociación con las administraciones. En esta investigación el factor se ha subdividido en dos constructos, Grado de Globalización de las empresas de un País (GLOBAL), Entorno Económico y Científico (EEC), debido a que el proceso de difusión/ adopción del conocimiento se ha visto acelerado por la apertura internacional y comercial de los países y, en segundo lugar, porque la globalización y el cambio tecnológico-científico están interrelacionados, reforzándose mutuamente. En este sentido Enkel et al. (2009) afirma al respecto que en el siglo XXI, los procesos de innovación/adopción son cada vez más abiertos y las empresas/países utilizan el conocimiento de fuentes externas de manera mucho más eficiente.

El factor Tecnológico representa a las tecnologías internas y externas relevantes para la firma que se encuentran accesibles, aunque sea en el mercado. En esta investigación a dicho factor se le ha denominado Contexto Tecnológico (TIC). En el factor empresarial se incluyen el tamaño de la empresa y ámbito; grado de centralización, formalización, complejidad de su estructura de gestión, cualificación de los recursos humanos; nivel de disponibilidad de los recursos. En esta investigación se corresponde con el constructo Capacidad de Gestión Empresarial del País para Adoptar ICT (CAGEP)

Y finalmente, se ha considerado como constructo dependiente a Adopción de Innovaciones ICT (ADOPT) en las empresas de cada país, entendiendo que cuantas más adopciones de ICT realice un país mejor situado se 
encuentra éste para acceder y utilizar ICT. Y cuanto mayor es la dimensión de los entornos tecnológico y de carácter empresarial mayor facilidad tiene un país para acceder a las ICT.

\section{MODELO TEÓRICO PROPUESTO}

Los vínculos y el sentido de las direcciones de los factores o constructos representan la relación de causalidad entre ellos. De acuerdo con las características del tema estudiado, las relaciones entre los constructos tendrían que ser bidireccionales pero hasta ahora los modelos SEM-PLS no lo permiten, así que se optó por un modelo unidireccional. Por consiguiente, a partir del análisis bibliográfico realizado se plantean diez hipótesis sobre las que se ha construido el modelo propuesto:

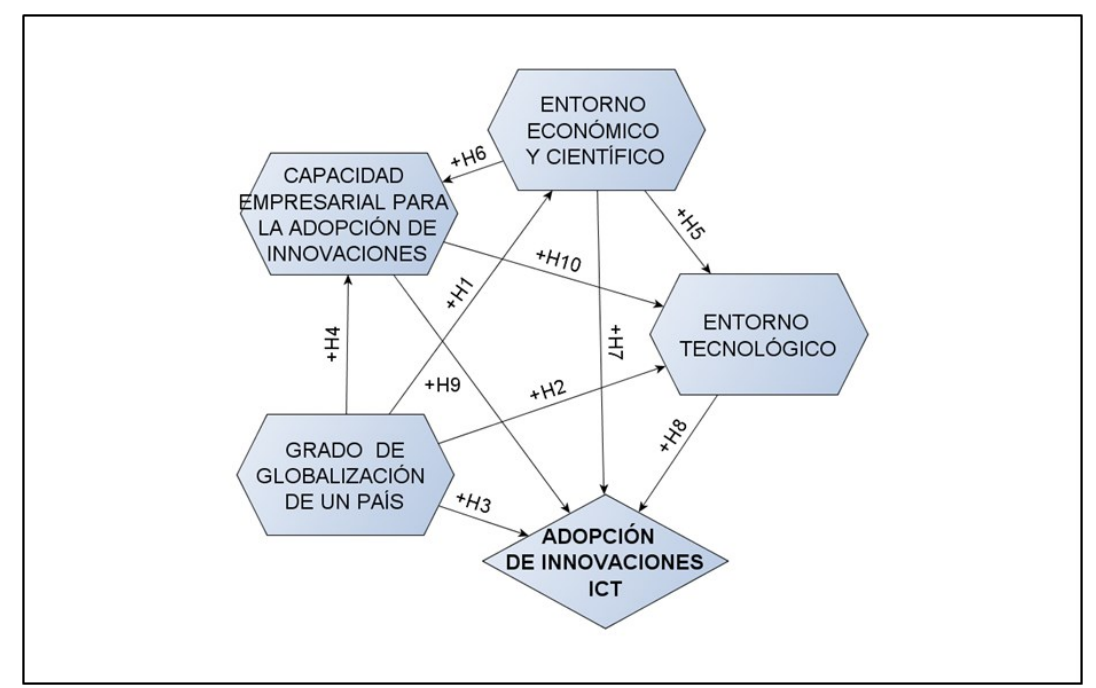

Figura 1 - Modelo de Investigación y desarrollo de hipótesis.

\section{Hipótesis}

Constructo GLOBAL.- El entorno global de cada país evoluciona de acuerdo con el volumen de facturación de las multinacionales fuera de su país de origen, con el valor total de la Inversión Directa Extranjera de las firmas de un país en el extranjero y en función del adquisición de conocimiento tecnológico a través de la Inversión directa Extranjera de tipo tecnológico (Palafox, 2017; Sahoo et al., 2013; Nunnenkamp and Spatz 2004; UNCTAD, 2013).

Pero, para que los países consigan desarrollar y mantener un entorno global significativo, las empresas internacionalizadas deben incrementar su conocimiento, desarrollar y aplicar ICT de forma constante (Gourlay and Pentecost, 2002; Bayo-Moriones and Lera-López, 2007; Wagner et al, 2003), usar intensivamente las redes informáticas y de telecomunicaciones (Vicente and López, 2011; Astrostic and Nguyen 2005), e incrementar la seguridad de los servidores de internet. Por lo tanto el constructo Global puede influir al constructo TIC.

Las multinacionales y las empresas internacionalizadas requieren desarrollar estructuras de organización y coordinación (Dyer y and Singh, 1998) para fidelizar al cliente, crear equipos multidisciplinares de I+D e innovación, generar vínculos duraderos con proveedores, etc para gestionar bien la innovación y la adopción tecnológica; por lo tanto, el constructo Global influye en el de CAGEP.

Así pues, cuanto mayor sea el tamaño de la red empresarial desde la perspectiva de la dimensión sectorial y geográfica mayor conocimiento tendrá la firma de las tecnologías disponibles en el mercado, y se podrá tomar la decisión de adoptar tecnología con mayor prontitud que otras firmas con una red más reducida (Abrahamson and Rosenkopf, 1997; Bayo-Moriones and Lera-López, 2007). Conviene tener en cuenta que la introducción de una firma en una red transnacional implica un reto de coordinación que solamente podrá ser superado mediante el empleo de tecnologías ICT, de ahí que las tecnologías ICT son un gran aliado en la expansión internacional de las empresas (Santangelo, 2001; Akmanligil, and Palvia, 2004).Así pues, el constructo GIOBAL puede influir en TIC. 
Por otra parte, estas empresas internacionalizadas necesitan encontrar en los países donde localizan plantas de producción, comercialización etc un EEC y un TIC bien dimensionados al objeto de que estos entornos favorezcan la adopción de ICT; dado que las infraestructuras digitales son críticas para integrar a los productores locales en las redes B2B y por ende atraer inversión directa extranjera (Addison and Heshmati, 2003).

Así pues, de acuerdo con esta explicación se plantea las siguientes hipótesis

H1.- El Constructo GLOBAL está positivamente relacionado con EEC.

H2.- El Constructo GLOBAL está positivamente relacionado con TIC.

H3.- El Constructo GLOBAL está positivamente relacionado con ADOPT.

H4.- El Constructo GLOBAL está positivamente relacionado con CAGEP.

Constructo EEC. El grado de desarrollo del entorno económico y científico de un país tiene una influencia positiva sobre la buena gestión de las empresas para adoptar innovaciones ICT y sobre el volumen de conocimiento y de infraestructuras ICT de un país. En este sentido, algunos autores (Blien and Maier, 2008; NietoAntolín y González- Álvarez, 2011) consideran que las políticas de los gobiernos para apoyar la disponibilidad de tecnologías ICT y la existencia de leyes y de normativas ajustadas al uso de dichas técnicas resultan fundamentales para las empresas y para la formación de entorno económico. De la misma manera que las adquisiciones gubernamentales de productos de tecnología avanzada impulsan el desarrollo del constructo TIC (Vicente and López, 2011). Todos estos elementos, junto con un índice elevado en la calidad de las escuelas de negocios crean confianza en las empresas y mejoran el rendimiento de las innovaciones ICT adoptadas.

Por lo tanto, en función de estos planteamientos se formula las siguientes hipótesis

H5.- El constructo EEC está positivamente relacionado con TIC

H6.- El constructo EEC está positivamente relacionado con CAGEP

H7.-El constructo EEC está positivamente relacionado con ADOPT

Constructo TIC. La disponibilidad de conocimiento e infraestructuras TIC varía entre países y resulta indispensable en la adopción de un producto o servicio ICT por parte de las empresas. El concepto de disponibilidad de tecnología (Bustamante-Donas, 2004) encierra el grado de difusión de las tecnologías ICT incluidas las infraestructuras, el número de adopciones de patentes ICT, y el grado de seguridad existente en los servidores de internet.

Pero, estas tecnologías para ser adaptadas (Gupta et al., 2013) deben ser fiables al objeto de que no presenten perdida de información, ofrezcan un servicio ininterrumpido y muestren condiciones de seguridad. De ahí que cuanto más importante sea el sector TIC y más seguras las infraestructuras ICT en una nación, más relevante puede ser la acumulación de conocimientos ICT, mayor el mercado de especialistas y la calidad de las infraestructuras ICT y, mayor será también la probabilidad de que las empresas de ese país adopten un producto o servicio ICT. Como consecuencia de ello, la posibilidad de que se produzcan externalidades de conocimiento ICT o spillovers se incrementará.

Así pues, en coherencia con este argumento se formula la siguiente hipótesis:

H8.- El constructo TIC se relaciona positivamente con ADOPT

Constructo CAGEP. El éxito de la buena gestión en la adopción de innovaciones ICT en las empresas obedece a la capacidad de innovación empresarial, al nivel de cualificación de los empleados en ICT (Chun 2003; HoIlenstein, 2004; Alderete, et al, 2014), a la facilidad de obtención de préstamos, a la naturaleza de la ventaja competitiva de las firmas y a la capacidad de organización (Brynjolfsson and Hitt ,2003). Lo que quiere decir que una empresa que cumpla con estas características dispone de recursos intangibles suficientes para gestionar la adopción de innovaciones ICT. Por lo tanto, la capacidad de una empresa para gestionar bien la adopción ICT influye en el nivel de desarrollo de TIC y su madurez, y como consecuencia, en el incremento de la tasa de adopción de ICT.

Pero, a pesar de las ventajas de las tecnologías ICT, el volumen de inversión en éstas, varía sustancialmente entre países, entre sectores y entre empresas. En este sentido, la evidencia sugiere que no todas las empresas de un país se apresuran a adoptar soluciones basadas en las tecnologías ICT (Trigueros- Preciado et al., 2013). Por ejemplo, la cloud computing es una tecnología punta que todavía tiene problemas por resolver (falta de normativa, alto nivel de riesgo, costes altos etc), y, donde su conocimiento por parte de las firmas es reducido (Oliveira et al., 2014).

Así pues, de acuerdo con estos razonamientos se plantean las siguientes hipótesis:

H9.- El constructo CAGEP se relaciona positivamente con ADOP

H10.- El constructo CAGEP se relaciona positivamente con TIC 
El constructo ADOPT representa la adopción de innovaciones ICT. Concretamente se compone de las variables B2B y el grado de presencia de M2M en el mercado. La primera representa la introducción de las ICT en las rutinas empresariales, mientras que la segunda sirve como proxy para valorar la comunicación Machine to Machine.

\section{METODOLOGÍA}

La escasez de fuentes estadísticas ha sido un factor a tener en cuenta en la realización de este trabajo, ya que la mayoría de la información disponible y, que además, fuese comparable internacionalmente se encuentra agregada a nivel de país. Ello obligó a adoptar a éste último como unidad de análisis, y a restringir el número de elementos de la muestra a la información disponible en estas fuentes. Por ello, dicha muestra está constituida por un conjunto de países desarrollados y por diversas economías en vías de desarrollo.

En este contexto, el modelo propuesto fue testado mediante una muestra construida a partir de cuatro fuentes estadísticas: World Competitiveness Report 2016, The Global Information Technology Report 2016, el informe Telecoms, Media and Entertainment Outlook de Ovum 2015 y Estadísticas World Bank 2016. Con la información de estas fuentes se elaboró una base de datos que consta de 18 variables, referidas a la gestión empresarial, entorno económico-científico, globalización empresarial y disponibilidad de tecnologías e infraestructuras TIC en el entorno.

La metodología empleada ${ }^{2}$ para el World Competitiveness Report y The Global Information Technology Report consiste en la realización de 14.000 entrevistas repetidas anualmente y dirigidas a directivos de corporaciones, distribuidas en 138 países. Por su parte World Bank ha realizado entre 1200 y 1800 entrevistas por país, dirigidas principalmente a propietarios y directivos de empresas. Finalmente se construyó una base de datos con 124 casos.

La información obtenida fue analizada mediante PLS-SEM posibilitando relacionar los constructos diseñados de una parte; y por otra, comprobar la validez de las sinergias causales identificadas con cada una de las hipótesis presentadas en el apartado anterior. Esta técnica resulta especialmente apropiada para abordar esta investigación debido al tamaño de la muestra empleada (Reinartz et al., 2009), y porque favorece el análisis simultaneo de los sistemas de variables.

Tras haber validado los constructos y los enlaces, es decir, la significatividad de la relación entre cada par de constructos del modelo se analiza éste último,( excluyendo los enlaces no significativos), como si fuera una red donde los constructos se consideran los vértices y los enlaces. Concretamente se realizan dos análisis. Por una parte, se estudia la influencia de los constructos en la red o modelo, y por otra, la transitividad de los factores dentro de esa red. El primer análisis consiste en la aplicación del algoritmo Beta Centrality, que valora la influencia de cada vértice a partir de la de sus vértices vecinos. El segundo algoritmo consiste en el análisis Betweenness Centrality, que valora la transitividad y por ende la influencia de cada constructo en la red.

\section{RESULTADOS}

Tras haber realizado la estimación del modelo, se han confirmado las hipótesis mediante el análisis de los path que relacionan los constructos (tabla 1). Para ello fue preciso analizar la significación de dichos path utlizando un bootstrap de 5000 muestras (Hair et al.; 2012)

De este modo los constructos CAGEP, TIC, GLOBAL, EEC explican un 85\% del constructo ADOPT, y, las hipótesis vinculadas a este último se han confirmado con una significatividad al $99 \% \mathrm{H} 8(\mathrm{p}<0.01), \mathrm{H} 3(\mathrm{p}<0.0)$, mientras que $\mathrm{H} 7$ ( $p<0.05)$ se confirmó a un $95 \%$ y $\mathrm{H} 9(\mathrm{p}<0.7)$ no resultó significativa.

El constructo CAGEP se explica en un $59 \%$ por los constructos GLOBAL y EEC y las hipótesis que los relacionan $\mathrm{H} 6$ y $\mathrm{H} 4$ respectivamente son significativas al $99 \% \mathrm{H} 6(\mathrm{p}<0.01)$ y $\mathrm{H} 4(\mathrm{p}<0.01)$.

El constructo TIC se explica en un 72\% por los constructos EEC, GLOBAL y CAGEP. Las relaciones con estos constructos han resultado significativas en todos los casos al 99\%, $\mathrm{H} 5(p<0.01), \mathrm{H} 2(p<0.01)$ y $\mathrm{H} 10(\mathrm{p}<0.01)$.

Y por último, el constructo EEC se explica en un 58\% por el constructo GLOBAL y la hipótesis que representa esta relación también ha resultado significativa $\mathrm{H} 1(\mathrm{p}<0.01)$.

\footnotetext{
${ }^{2}$ Del Informe Telecoms, Media and Entertainment Outlook de Ovum de 2015 no se conoce la metodología empleada.
} 
Tabla 1 - Coeficiente y significatividad de cada relación o hipótesis.

\begin{tabular}{|c|c|c|c|}
\hline HIPOTESIS & DEFINICIÓN & $\begin{array}{c}\text { COEFICIENTES } \\
\text { DE LOS ENLACES }\end{array}$ & $\mathrm{t}$-student \\
\hline $\mathrm{H} 9$ & CAGEP $\rightarrow$ ADOPT & 0.05 & 0.74 \\
\hline $\mathrm{H} 8$ & TIC $\rightarrow$ ADOPT & 0.63 & $8.83^{* * *}$ \\
\hline H5 & EEC $\rightarrow$ TIC & 0.32 & $3.29^{* * *}$ \\
\hline H6 & EEC $\rightarrow$ CAGEP & 0.51 & $5.84^{* * *}$ \\
\hline H1 & GLOBAL $\rightarrow$ EEC & 0.73 & $17.44^{* * *}$ \\
\hline H4 & GLOBAL $\rightarrow$ CAGEP & 0.33 & $3,78^{* * *}$ \\
\hline H2 & GLOBAL $\rightarrow$ TIC & 0.24 & $2,42^{* * *}$ \\
\hline H7 & EEC $\rightarrow$ ADOPT & 0.13 & $1.99^{* *}$ \\
\hline H3 & GLOBAL $\rightarrow$ ADOPT & 0.16 & $3.02^{* * *}$ \\
\hline H10 & CAGEP $\rightarrow$ TIC & 0.35 & $4.73^{* * *}$ \\
\hline
\end{tabular}

Los asteriscos, ${ }^{*},{ }^{* *}, \mathrm{y}^{* * *}$ representan la significatividad al $90 \%, 95 \%$, and $99 \%$, respectivamente. Fte: Elaboración propia a partir de las bases de datos

La intensa relación habida entre los constructos que componen el modelo indujo a pensar, de una parte, en la existencia de efectos indirectos en las relaciones de los constructos, y, de otra, que éstos contribuyen a dar explicaciones ulteriores del papel de los constructos en la adopción de innovaciones se muestran en la tabla 2.

En el modelo propuesto se han encontrado hasta 7 posibles efectos indirectos de los constructos GLOBAL, EEC y CAGEP sobre ADOPT (tabla 2). En todos ellos, excepto en uno se han constatado mediaciones parciales, se trata pues de la mediación con origen en CAGEP que es total.

La agregación de los coeficientes de los enlaces indirectos (tabla 2) según origen ha permitido obtener los constructos con mayor influencia en ADOPT. Éstos son: $\operatorname{GLOBAL}(0,4)$, EEC $(0,3)$ y CAGEP $(0,2)$.Por lo tanto, GLOBAL tiene mucha mayor influencia indirecta en el modelo que directa (0,48 indirecto frente a 0,16 directo). Algo similar ocurre con $\operatorname{EEC~}(0,3)$ de efecto indirecto frente a 0,13 directo y especialmente con CAGEP $(0,05)$ efecto directo frente a 0,2 indirecto. De ello se concluye que la influencia indirecta de estos constructos sobre la adopción de innovaciones es mucho mayor que la directa.

Tabla 2 - Análisis de los efectos indirectos de los constructos.

\begin{tabular}{|c|c|c|c|c|c|c|c|c|c|}
\hline \multirow[b]{2}{*}{ ORIGEN } & \multirow{2}{*}{ Mediación } & \multicolumn{3}{|c|}{ Efectos directos } & \multicolumn{3}{|c|}{ Efectos indirectos } & \multirow{2}{*}{ Tipo $^{1}$} & \multirow{2}{*}{ Efectos totales } \\
\hline & & Enlac. & sig. & $+/-$ & Enlac. & sig & $+/-$ & & \\
\hline GLOBAL & $\mathrm{H} 1 \rightarrow \mathrm{H} 7$ & \multirow{4}{*}{0.16} & 99 & + & 0.09 & 90 & + & MP & \multirow{4}{*}{0.64} \\
\hline GLOBAL & $\mathrm{H} 1 \rightarrow \mathrm{H} 5 \rightarrow \mathrm{H} 8$ & & 99 & + & 0,15 & 99 & + & MP & \\
\hline GLOBAL & $\mathrm{H} 2 \rightarrow \mathrm{H} 8$ & & 99 & + & 0.15 & 95 & + & MP & \\
\hline GLOBAL & $\mathrm{H} 1 \rightarrow \mathrm{H} 6 \rightarrow \mathrm{H} 10 \rightarrow \mathrm{H} 8$ & & 99 & + & 0.09 & 99 & + & MP & \\
\hline EEC & $\mathrm{H} 5 \rightarrow \mathrm{H} 8$ & \multirow[t]{2}{*}{0.13} & 95 & + & 0.2 & 99 & + & MP & \multirow{2}{*}{0.43} \\
\hline EEC & $\mathrm{H} 6 \rightarrow \mathrm{H} 10 \rightarrow \mathrm{H} 8$ & & 95 & + & 0.1 & 99 & + & MP & \\
\hline CAGEP & $\mathrm{H} 10 \rightarrow \mathrm{H} 8$ & 0.05 & no & + & 0.2 & 99 & + & MT & 0.25 \\
\hline TIC & $\mathrm{H} 8$ & 0.63 & 99 & + & - & - & - & SM & 0.63 \\
\hline
\end{tabular}

${ }^{1}$ Tipo de mediación: M.P. (Mediación parcial), M.T. (Mediación total), S.M. (Sin mediación).

Fte: Elaboración propia a partir de las bases de datos citadas.

La estimación del algoritmo Beta Centrality (tabla 3) presenta el orden de influencia de los constructos en la red, siendo éste similar a los del análisis anterior. Así pues, el orden de los constructos influyentes en la red es: GLOBAL $(4,30)$, EEC $(2,2)$, TIC $(0,9)$ y CAGEP $(0,8)$; mientras que ADOP no puede tener influencia al ser un mero receptor.

Por último, las estimaciones del algoritmo Betweenness (tabla 3) para cada constructo ha evidenciado al constructo TIC como un transmisor esencial. De todo ello se puede concluir que aunque los constructos más influyentes sobre ADOP son GLOBAL y EEC, su influencia será inexistente si el constructo TIC obtiene un valor de 0 . Para que exista influencia indirecta de los primeros, el constructo TIC debe de tener unos valores mínimos. 
Tabla 3 - Análisis de redes del modelo testado.

\begin{tabular}{|c|c|c|}
\hline & Betweenness & Beta centrality \\
\hline GLOBAL & 0,0 & 4,3 \\
\hline EEC & 0,0 & 2,2 \\
\hline CAGEP & 0,0 & 0,8 \\
\hline TIC & 0,08 & 0,9 \\
\hline ADOPT & 0,0 & 0,0 \\
\hline
\end{tabular}

Fte: Elaboración propia a partir de las bases de datos citadas.

\section{CONCLUSIONES}

La modelización del proceso de innovaciones ICT mediante PLS-SEM, ha constatado:

1.- Que los constructos que más inciden en la adopción de innovaciones ICT fueron GLOBAL debido a la influencia indirecta de éste en el modelo. El constructo del entorno tecnológico ICT (TIC) por su gran influencia sobre la adopción de innovaciones, así como por su papel transmisor de los efectos indirectos de los demás constructos. Y el constructo entorno económico y científico de los países (EEC) por su carácter estratégico dado que es fácilmente manipulable por los estados.

2.- La modelización del proceso de innovaciones ICT ha comprobado la existencia de relaciones de causalidad entre los constructos explicativos, lo que a su vez ha facultado a realizar un análisis de los efectos indirectos de dichos constructos explicativos sobre ADOPT. Tras validar las relaciones entre constructos y comparar la influencia directa e indirecta de éstos sobre ADOPT, se observó que los indirectos son más importantes que los primeros, para la adopción de innovaciones ICT (0,98 en los enlaces directos, frente a 1,021 en los indirectos). Dentro de los efectos directos destaca sobre todos los demás el constructo TIC, mientras que en los indirectos sobresalen GLOBAL y CESE.

En relación a éste último cabe constatar que hasta ahora las investigaciones realizadas a nivel de empresa han prestado poco interés al impacto de las instituciones públicas y privadas sobre la adopción de ICT, a la regulación de éstas últimas y a la influencia de las instituciones que asisten a empresas creando soluciones de ingeniería adaptadas a sus necesidades. Igualmente, la atención de la literatura científica sobre la globalización ha sido más bien escasa, cuestión que llama la atención porque las firmas que se inician en el proceso de globalización y de internacionalización presentan una gran necesidad de adoptar tecnologías ICT que les permita realizar su tarea productiva coordinada en tiempo real; y gestionar, al mismo tiempo, mediante el empleo de TICs un enorme volumen de información. De la misma manera, la existencia de conocimiento ICT accesible en el país a través de rutinas empresariales, equipos TIC integrados en la producción, y la codificación de conocimiento tácito mediante patentes y diseños, es un factor determinante en la canalización de todas las influencias positivas de la globalización y las instituciones en la adopción de tecnologías ICT.

Sin embargo, la influencia de estos dos constructos sobre TIC puede ser unidireccional o recíproca, en este sentido, resulta conveniente mencionar la limitación que ha supuesto la aplicación del modelo PLS-SEM que solo puede establecer relaciones de causalidad unidireccionales. La relación entre el factor TIC y el factor GLOBAL es más bien recíproca, es decir, la existencia de conocimiento ICT posibilita globalizarse, pero a su vez, la globalización de las actividades económicas demanda crecientemente métodos de coordinación de ventas y producción más eficientes. Asimismo, la apertura de una empresa en otros mercados posibilita el conocimiento y adopción de tecnologías ICT no conocidas en el entorno nacional. Igualmente, el factor EEC es reciproco en lo que respecta a las empresas del entorno, especialmente cuando éstas se encuentren en la cadena proveedor cliente de la empresa. Sin embargo, la influencia del entorno institucional es más bien unidireccional, puesto que las instituciones de conocimiento suelen estar enfocadas a auxiliar la actividad productiva y no al revés. Así pues, en futuros trabajos se podría modelizar las relaciones entre los constructos en sentido inverso, a fin de estudiar las relaciones no modelizadas entre los factores explicativos de esta investigación.

Entre las principales implicaciones empresariales e institucionales de este trabajo sobre la adopción de ICT cabe destacar que el fomento de la adopción de innovaciones ICT en empresas debería contemplar la estimula- 
ción de los factores más influyentes en la adopción: la internacionalización de las firmas considerando particularmente la transferencia tecnológica, y el desarrollo del conocimiento tácito y explicito empresarial e institucional en las tecnologías ICT. Éste último es estratégico, por un lado, por ser el más influyente sobre ADOPT; y, por otro, porque es el principal canalizador de los efectos indirectos de los factores estudiados. En este sentido, es preciso tener presente que para que la globalización o las instituciones tengan influencia en el incremento de conocimientos ICT es conveniente que este factor no sea nulo o inexistente. De este modo, si la demanda privada no es capaz de desarrollar competencias ICT en el territorio accesibles a todo el territorio nacional, sería conveniente favorecer desde instancias públicas mediante el esfuerzo de las instituciones que incrementen la producción de conocimiento ICT (patentes, software, etc), el fomento de legislación en materia de ICT y el establecimiento de criterios de contratación pública afines a la innovación y la adopción de ICT.

\section{REFERENCES}

ABRAHAMSON, E. AND ROSENKOPF, L. (1997), "Social network effects on the extent of innovation diffusion: A computer simulation", Organization science, Vol. 8 No. 3, pp. 289-309.

ADDISON, T. AND HESHMATI, A. (2003). The new global determinants of FDI flows to developing countries: The importance of ICT and democratization (No. 2003/45). WIDER Discussion Papers//World Institute for Development Economics (UNUWIDER).

AKMANLIGIL, M. AND PALVIA, P. C. (2004), "Strategies for global information systems development", Information \& Management, Vol. 42 No. 1, pp. 45-59.

ALDERETE, M. V., JONES, C. AND MORERO, H. A. (2014), "Factores explicativos de la adopción de las TIC en las tramas productivas automotriz y siderúrgica de Argentina", Pensamiento \& Gestión, Vol. 37, pp. 1-40.

ARMBRUST, M., FOX, A., GRIFFITH, R., JOSEPH, A. D., KATZ, R., KONWINSKI, A. AND ZAHARIA, M. (2010), "A view of cloud computing", Communications of the ACM, Vol. 53 No. 4, pp. 50-58.

BAYO-MORIONES, A. AND LERA-LÓPEZ, F. (2007), "A firm-level analysis of determinants of ICT adoption in Spain", Technovation, Vol. 27 Issue 6, 352-366.

BACH, M. P., ZOROJA, J., Y VUKŠI, V. B. (2013), "Determinants of firms' digital divide: A review of recent research, Procedia Technology", Vol 9, pp. 120-128.

BLIEN, U. AND MAIER, G. (2008), The Economics of Regional Clusters: Networks, Technology and Policy, Edward Elgar Publisihing, London.

BRYNJOLFSSON, E., AND HITT, L. M. (2000), "Beyond computation: Information technology, organizational transformation, and business performance", Journal of Economic Perspectives, Vol. 14 No. 4, pp. 23-48.

BUSTAMANTE-DONAS, J. (2004), "¿Qué puede esperar la democracia de internet? una reflexión sobre la crítica de Langdon Winner al poder político transformador de la tecnología, Argumentos de Razón Técnica", Revista Española de Ciencia, Tecnología y Sociedad y Filosofía de la Ciencia, No.7, pp. 13-49.

BUYYA, R., YEO, C. S., VENUGOPAL, S., BROBERG, J. AND BRANDIC, I. (2009), "Cloud computing and emerging IT platforms: Vision, hype, and reality for delivering computing as the 5th utility", Future Generation computer systems, vol. 25 No. 6, pp. 599-616.

CAPÓ-VICEDO, J., TOMÁS-MIQUEL, J. AND EXPÓSITO-LANGA, M. (2007), "La gestión del conocimiento en la cadena de suministro. Análisis de la influencia del contexto organizativo", Revista Información Tecnológica, Vol. 18 No. 1, pp. 127-134

CHUN, H. (2003). "Information Technology and the Demand for Educated Workers: Disentangling the Impacts of Adoption versus Use". The Review of Economics and Statistics, Vol. 85 No. 1, pp. 1-8.

DYER, J. AND SINGH, H. (1998), "The Relational View: Cooperative Strategy and Sources of Interorganizational Competitive Advantage", Academy of management, Vol. 23 No. 4, pp. 660-679.

ENKEL, E., GASSMANN, O. AND, H. (2009), "Open R\&D and open innovation: exploring the phenomenon", R\&d Management, Vol. 39 No.4, pp. 311-316.

FRENZ, M. AND LETTO-GILLIES, G. (2009), "The impact on innovation performance of different sources of knowledge: Evidence from the UK Community Innovation Survey". Research Policy, No. 38, pp. 1125-1135

GERTLER, M. S. (2003), "Tacit knowledge and the economic geography of context, or the undefinable tacitness of being (there)", Journal of economic geography, Vol. 3. No. 1, pp. 75-99. 
GUPTA, P., SEETHARAMAN, A. AND RAJ, J. R. (2013), "The usage and adoption of cloud computing by small and medium businesses", International Journal of Information Management, Vol. 33 No. 5, pp. 861-874.

GOURLAY, A., AND PENTECOST, E. (2002), "The determinants of technology diffusion: Evidence from the UK financial sector", The Manchester School, Vol. 70 No. 2, pp.185-203.

HAIR, J. F., SARSTEDT, M., RINGLE, C. M. AND MENA, J. A. (2012), "An assessment of the use of partial least squares structural equation modeling in marketing research". Journal of the academy of marketing science, Vol. 40 No. 3, pp. $414-433$.

HAYES, A. F. AND SCHARKOW, M. (2013), "The relative trustworthiness of inferential tests of the indirect effect in statistical mediation analysis does method really matter?". Psychological science, Vol. 24 No. 10, pp. 1918-1927.

HOLLENSTEIN, H. (2004). "Determinants of the adoption of Information and Communication Technologies (ICT): An empirical analysis based on firm-level data for the Swiss business sector". Structural change and economic dynamics, Vol. 15 No. 3 , pp. 315-342.

HONG, W. AND ZHU, K. (2006), "Migrating to internet-based e-commerce: Factors affecting e-commerce adoption and migration at the firm level". Information \& Management, Vol. 43 No. 2, pp. 204-221.

HOTI, E. (2015), "The technological, organizational and environmental framework of IS innovation adaption in small and medium enterprises. Evidence from research over the last 10 years". International Journal of Business and Management, Vol. 3 No. 4, pp. 1-14.

IFINEDO, P. (2011), "An empirical analysis of factors influencing Internet/e-business technologies adoption by SMEs in Canada", Journal and International Technology and Decision Making, Vol. 10, No. 4, pp. 19-28.

LAURSEN, K. AND SALTER, A. (2006), "Open for innovation: the role of openness in explaining innovation performance among UK manufacturing firms". Strategic Management Journal, Vol. 27 No. 2, pp. 131-150.

LAURSEN, K., REICHSTEIN, T. AND SALTER, A. (2011), "Exploring the effect of geographical proximity and university quality on university-industry collaboration in the United Kingdom", Regional studies, Vol. 45 No. 4, pp. 507-523.

LIN, H. F. AND LIN, S. M. (2008), "Determinants of e-business diffusion: A test of the technology diffusion perspective", Technovation, Vol. 28 No. 3, pp. 135-145.

MAYER-SCHÖNBERGER, V. AND CUKIER, K. (2013), Big data: A revolution that will transform how we live, work, and think, Houghton Mifflin Harcourt, New York.

MILBERG, W. AND WINKLER D. (2013) Outsourcing economics: global value chains in capitalist development. Cambridge university press.

NIETO-ANTOLÍN, M. AND GONZÁLEZ-ÁLVAREZ, N. (2011), "Estructura de la industria, entorno institucional y actividad innovadora en la empresa industrial Española", ICE, No. 860, pp. 57-71.

NUNNENKAMP, P. AND SPATZ, J. (2004), "FDI and Economic Growth in developing Economies: How relevant are host economy and industry characteristics, Transnational Corporations, Vol. 13, No. 3, pp 54-86

OLIVEIRA, T., THOMAS M., AND ESPADAÑAL, M. (2014), "Assessing the determinants of cloud computing adoption: An analysis of the manufacturing and services sectors", Revista Information and Management, Vol. 51, pp. $497-510$.

OLIVEIRA, T. AND MARTIN, M. F. (2010), "Firms patterns of e-business adoption: evidence for the European Union-27". The 